Abstract

\title{
Generalization of the Noether Theorem: Global and Local Symmetries, Dynamical Functionals and Boundary Conditions ${ }^{\dagger}$
}

Plamen Fiziev 1,2

1 Joint Institute for Nuclear Research (JINR), 141980 Dubna, Russia; fiziev@theor.jinr.ru

2 Foundation of Theoretical and Computational Physics and Astrophysics, Sofia 1164, Bulgaria

* Correspondence:

+ Presented at Symmetry 2017-The First International Conference on Symmetry, Barcelona, Spain, 16-18 October 2017.

Published: 4 January 2018

\begin{abstract}
We consider the most general form of the Noether theorem which is suitable for the treatment of global symmetries, described by symmetry-groups, and local symmetries, described by symmetry-algebras, which may not be integrable to symmetry groups. The principle new notion is specific dynamical functionals which solve the problem by replacing the standard action functionals. We also discuss the compatibility of the boundary conditions and the corresponding variational principles. Many illustrative examples are shown.
\end{abstract}

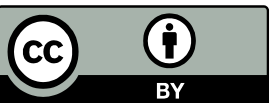

(C) 2018 by the author. Licensee MDPI, Basel, Switzerland. This article is an open access article distributed under the terms and conditions of the Creative Commons Attribution (CC BY) license (http://creativecommons.org/licenses/by/4.0/). 
